Revista de Matemática: Teoría y Aplicaciones 2012 19(2) : 211-237

CIMPA - UCR ISSN: $1409-2433$

\title{
APLICACIÓN DE UN MODELO COGNITIVO DE VALORACIÓN EMOTIVA A LA FUNCIÓN DE EVALUACIÓN DE TABLEROS DE UN PROGRAMA QUE JUEGA AJEDREZ
}

\author{
APPLICATION OF A COGNITIVE MODEL OF \\ EMOTIONAL APPRAISAL TO THE BOARD \\ EVALUATION FUNCTION OF A PROGRAM THAT \\ PLAYS CHESS \\ Ana Lilia Laureano-Cruces* \\ Diego EnRIQue HeRnÁNDEZ-GonZÁLEZ ${ }^{\dagger}$ \\ Martha Mora-Torres ${ }^{\ddagger} \quad J_{\text {Javier RAmírez-RodríGuez }}^{\S}$
}

Received: 3 May 2010; Revised: 13 Dec 2011;

Accepted: 10 May 2012 


\title{
Resumen
}

En este trabajo se describe el análisis y diseño de un modelo cognitivo de valoración emotiva. Dicho modelo está basado en las metas que busca un jugador de ajedrez durante una partida. Este modelo afecta la salida de la función de evaluación de tableros de un agente computacional que juega ajedrez; dándole más peso a aspectos relacionados con las metas más urgentes; de acuerdo al contexto. De esta manera, la percepción del entorno cambia, y en consecuencia, la elección de la siguiente jugada. El objetivo es que el agente computacional incorpore en el proceso de toma de decisiones el estado emotivo y elija las jugadas con base en dicho estado. Para lograrlo se utilizaron herramientas de psicología cognitiva y computación suave con el fin de simular la emoción, y afectar el comportamiento del agente computacional.

Palabras clave: ajedrez computacional; modelos mentales; valoración emotiva; emociones sintéticas; función de evaluación; mapas cognitivos difusos

\begin{abstract}
This paper describes the analysis and design of a cognitive emotional assesment model. This model is based on the goals looked for a chess player during a game. The model affects the output of the board evaluation function of a computational agent which plays chess, giving more weight to aspects related to the most urgent goals, according to the context. Thus, it perceives environment changes, and consequently changes its choice of the next play. The aim is that the computational agent incorporates into the decision making process, the emotional state and choose the plays based on that state. For that purpose were used tools of cognitive psychology and soft computing in order to simulate emotion, and affect the agent's behavior computer.
\end{abstract}

Keywords: computer chess; mental models; emotive elicitation; synthetic emotions; evaluation function; fuzzy cognitive maps

Mathematics Subject Classification: 68T20, 68T37.

\footnotetext{
*Departamento de Sistemas, Universidad Autónoma Metropolitana-Azcapotzalco, Avenida San Pablo 180, 02200 México D.F., México. Laboratoire d'Informatique d'Avignon Université d'Avignon et des Pays de Vaucluse, France. E-Mail: clc@correo.azc.uam.mx

${ }^{\dagger}$ Posgrado en Ciencia e Ingeniería de la Computación, IIMAS-UNAM, Circuito Escolar, Ciudad Universitaria, 04510 México D.F., México. E-Mail: di_hernandez@uxmcc2.iimas.unam.mx

${ }^{\ddagger}$ Posgrado en Ciencia e Ingeniería de la Computación, IIMAS-UNAM, same address as Hernández-González. E-Mail: kabhun@yahoo.com.mx

${ }^{\S}$ Departamento de Sistemas, Universidad Autónoma Metropolitana-Azcapotzalco, same address as Laureano-Cruces. Laboratoire d'Informatique d'Avignon Université d'Avignon et des Pays de Vaucluse, France. E-Mail: jararo@correo.azc.uam.mx
}

Rev.Mate.Teor.Aplic. (ISSN 1409-2433) Vol. 19(2): 211-237, July 2012 


\section{Introducción}

Durante una partida de ajedrez, los jugadores están sometidos a estrés. Este juego es competitivo en gran medida, incluso los jugadores aprenden a manejar sus emociones durante las partidas [14] y aquellos que desarrollan en mayor medida esta habilidad son los que juegan mejor [5]. Este estrés podría influir en la manera en la que los ajedrecistas eligen jugadas.

Se ha encontrado que las emociones tienen un impacto considerable en el proceso de toma de decisiones, que el déficit en la capacidad de experimentar emociones degrada al proceso de toma de decisiones y que la incorporación de las emociones a los modelos de toma de decisiones aumenta su capacidad descriptiva [28].

Dos efectos de las emociones sobre el individuo son la reorganización de su jerarquía de metas y el enfoque de su atención en aspectos de su entorno en función de esas metas $[1,2,9]$. Cuando un individuo experimenta una emoción, su jerarquía de metas se restablece, colocando la más urgente en primer lugar; la importancia de una meta está en función del contexto del individuo y sus experiencias previas. Por otro lado, su atención se enfoca sobre las variables del entorno que son relevantes para el cumplimiento de esa meta; los demás aspectos son discriminados $[1,2]$.

Esas variables relevantes adquieren mayor peso durante el proceso de toma de decisiones, orientándolo hacia el cumplimiento de la meta más urgente. En [3] se expone un ejemplo de este efecto: las personas que están excitadas sexualmente buscan satisfacer su necesidad (la meta urgente) e ignoran factores de su contexto que podrían afectar otras metas (e. g. el riesgo de contraer una enfermedad de transmisión sexual o el violar tabúes y normas sociales) en el cumplimiento de su meta principal. Este efecto también está relacionado con la deformación de la percepción hacia las consecuencias esperadas al tomar cierta decisión: se les da mayor peso a aspectos relacionados con metas urgentes [29].

Este trabajo plantea cómo implementar un agente computacional que juegue ajedrez, centrado en lo que sentiría un jugador al observar determinadas posiciones de las piezas en el tablero $[11,12,13]$.

Los programas y sistemas de ajedrez que toman en cuenta el aspecto emocional del ajedrez son pocos y están orientados a la interacción humanocomputadora [6, 17, 27, 42].

El artículo está organizado de la siguiente manera: la sección 2 introduce la función de evaluación del tablero de ajedrez y los parámetros incluidos en esta función; la sección 3 expone cómo se desarrolló el modelado de la valoración emotiva desde la perspectiva del jugador y cómo se representó dicho modelo a través de un mapa cognitivo difuso; la sección 4 explica cómo se modifican los pesos de los parámetros de la función 


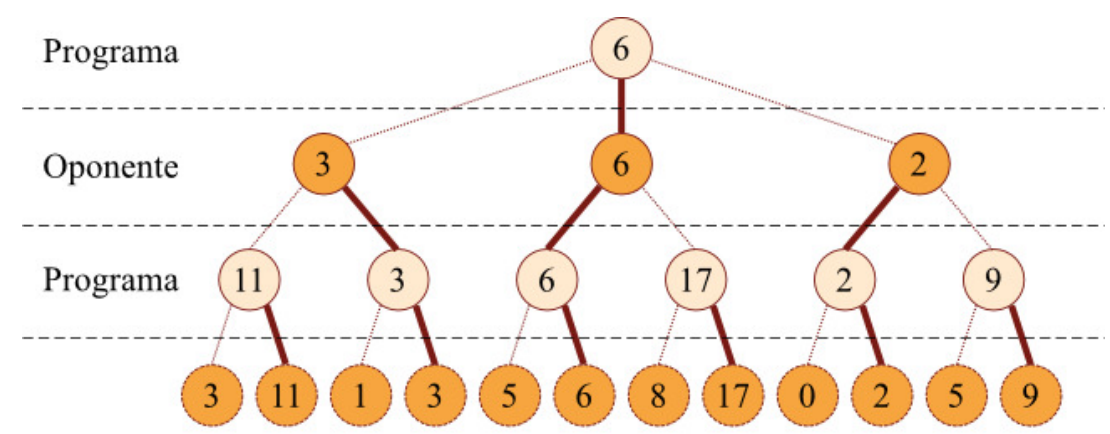

Nodos terminales evaluados

Figura 1: Árbol de exploración de juego.

de evaluación a partir de la salida del modelo de valoración emotiva; la sección 5 muestra un ejemplo del funcionamiento del sistema a partir del instante previo a la elección de una jugada; finalmente la sección 6 expone las conclusiones y líneas de investigación futura.

\section{Evaluación del tablero de ajedrez}

\subsection{Estrategia usada en la implementación de programas de ajedrez por computadora}

La estrategia más usada en la implementación de estos programas fue introducida en [44]. En el turno de mover del programa de ajedrez, a partir de la posición del tablero en un instante, se construye un árbol de juego de altura limitada. En esta estructura de datos están representadas jugadas legales y posiciones resultantes de dichas jugadas: cada rama del árbol representa una jugada y cada nodo una posición del tablero (Figura $1)$.

Tanto las jugadas del programa de ajedrez como las del oponente están representadas en dicho árbol y se van alternando entre niveles.

Después de obtener el árbol, se ejecuta un algoritmo de búsqueda entre adversarios para encontrar la jugada que maximice la ganancia de utilidad para el programa. De manera recursiva, en cada nodo, el algoritmo de búsqueda elige al nodo hijo con la utilidad más favorable para el programa, y devuelve ese valor de utilidad al nodo padre. Si se está evaluando un nodo terminal del árbol, entonces el algoritmo mide la utilidad de la posición del tablero correspondiente a ese nodo mediante una función de evaluación. La función de evaluación del tablero consiste en la suma de un conjunto de parámetros de evaluación que se pueden medir en la posición del tablero [30]. En el nodo raíz, se elige la rama que lleva al nodo hijo con la utilidad 
más deseable, y la jugada representada por esa rama es la que se ejecuta.

\subsection{Parámetros de evaluación del tablero}

Definimos como parámetro de evaluación a cualquier aspecto de la posición del tablero que sea necesario conocer para evaluar si la partida está a favor o en contra. Cuando se aplica la función de evaluación a una posición del tablero, se mide o se determina un valor a cada parámetro, y posteriormente se les asigna un peso de acuerdo a la importancia que tengan con base en la elección de la jugada siguiente [8]. En este proyecto se tomarán en cuenta los siguientes parámetros ya que son considerados como los más relevantes en la evaluación del tablero $[8,10,38]$ :

- Balance de material. Es la ventaja en cantidad y fortaleza de las piezas presentes en el tablero con respecto al oponente. Este parámetro se mide usualmente en centipeones (cp), una unidad que es equivalente a la centésima parte del valor asignado a un peón. En este proyecto, los valores materiales asignados a cada pieza son los siguientes: peón, $100 \mathrm{cp}$; caballo, $325 \mathrm{cp}$; alfil, $325 \mathrm{cp}$; torre, $500 \mathrm{cp}$; reina, $975 \mathrm{cp}$. Estas magnitudes fueron encontradas en un análisis estadístico [4].

- Movilidad. Se refiere al número de movimientos legales disponibles en la posición presente [46].

- Seguridad del rey e indefensión del rey del oponente. La seguridad del rey es una estimación de la protección de esta pieza en contra de los ataques del oponente. En este proyecto se incluye la evaluación de la seguridad del rey del oponente, al cual se denominó indefensión del rey del oponente. La seguridad del rey se evalúa de diversas formas, no existe un consenso único de cómo medir este parámetro. Para este trabajo, se eligieron las siguientes:

- Escudo de peones. Es una bonificación o penalización asignada por cada peón que se encuentre protegiendo o no al rey.

- Reytropismo. Es una estimación del riesgo de jaque al rey. No es que el riesgo aumente conforme más cerca esté una pieza del rey, sino que este parámetro incrementa la probabilidad de que el programa pueda elaborar un ataque. Dicha estimación es inversamente proporcional a la distancia entre las piezas contrarias y el rey. 


\subsection{Estimación numérica de los parámetros de evaluación}

La estimación de los parámetros de evaluación se basó en la manera propuesta por la función de evaluación del motor de ajedrez de Chess Programming Wiki (CPW). El código fuente de este motor se puede consultar en la siguiente página web: http://www.marittima.pl/cpw.

El balance de material se determina por medio de la ecuación (1):

$$
B=\sum_{i=1}^{n} v_{i}-\sum_{j=1}^{m} v_{j},
$$

donde:

- $v$ es el valor de una pieza presente en el tablero; $v_{i}$ es una pieza que pertenece al programa y $v_{j}$ es una pieza que pertenece al oponente,

- $n$ es el número de piezas que tiene el programa,

- $m$ es el número de piezas que tiene el oponente.

La movilidad se determina por medio de la ecuación (2):

$$
M=\sum_{i=1}^{n} m_{i},
$$

donde:

- $m_{i}$ es el número de casillas adonde se puede mover la pieza $i$,

- $n$ es el número de piezas presentes en el tablero que pertenecen al programa.

La seguridad del rey se determina por medio de la ecuación (3):

$$
S=h-r,
$$

donde:

- $S$ es la seguridad del rey,

- $h$ es la bonificación o penalización asignada al escudo de peones,

- $r$ es la magnitud del reytropismo hacia el rey.

El escudo de peones se determina por medio de la ecuación (4):

$$
h=8 p_{2 f}+4 p_{3 f}-8 c_{a b},
$$

donde: 
- $p_{2 f}$ es el número de peones que se encuentran en la segunda fila, delante de rey,

- $p_{3 f}$ es el número de peones en la 3 fila, delante del rey,

- $c_{a b}$ es el número de columnas abiertas junto al rey.

El reytropismo está determinado por la ecuación (5):

$$
r=\sum_{i=1}^{n}\left(14-\frac{\left(\left\|c_{0}-c_{i}\right\|+\left\|f_{0}-f_{i}\right\|\right)}{2}\right) w_{i}+p_{\text {alfil }}+p_{\text {torre }}
$$

donde:

- $c_{0}$ es la columna en donde está colocado el rey,

- $c_{i}$ es la columna en donde está colocada la pieza contraria $i$,

- $f_{o}$ es la fila en donde está colocado el rey,

- $f_{i}$ es la fila en donde está colocada la pieza contraria $i$,

- $w_{i}$ es un factor de peso en función del tipo de la pieza contraria,

- $p_{a l f i l}$ es una penalización que se calcula si la pieza contraria $i$ es un alfil,

- $p_{\text {torre }}$ es una penalización que se estima si la pieza contraria $i$ es una torre.

El factor de peso $w_{i}$ es igual a:

$$
w_{i}= \begin{cases}5 / 2 ; & p_{i}=\text { reina } \\ 1 / 2 ; & p_{i}=\text { torre, alfil } \\ 1 ; & p_{i}=\text { caballo }\end{cases}
$$

La penalización del alfil es igual a 3, si el rey se encuentra en la misma diagonal; 2 , si se encuentra a una diagonal de distancia; 1 , si se encuentra a 2 diagonales de distancia. La penalización de la torre es igual a 4, si se encuentra en una columna abierta junto al rey. La indefensión del rey del oponente se estimará de la misma manera que la seguridad del rey, pero con el signo contrario. 


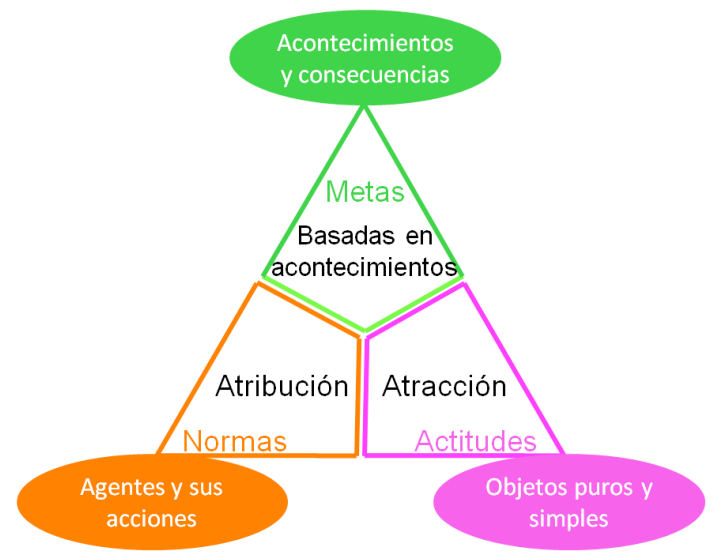

Figura 2: Estructura emocional; teoría OCC [22].

\section{Modelado de la valoración emotiva}

\subsection{Teoría cognitiva de emociones OCC}

El modelo de valoración emocional determina, para la posición en curso en el tablero (estado del tablero), la intensidad de la reacción emotiva dado ese estado. Este está basado en la teoría cognitiva de emociones OCC (Ortony, Clore, Collins) [40], cuya metodología permite evaluar la posible emoción desde el punto de vista cognitivo. Debido a esta metodología clara, precisa y libre de contexto que la identifica, la teoría OCC ha sido utilizada de forma frecuente en la síntesis de emociones por computadora [41, pág. 196].

Esta teoría propone una estructura general en la que se especifica que existen tres grandes clases de emociones, resultado de centrarse sobre cada uno de los tres aspectos destacados del mundo (Figura 2): 1) acontecimientos y sus consecuencias, 2) agentes y sus acciones y 3) objetos puros y simples. Para ello establece como criterios de valoración: 1) la importancia de las metas dentro de la macro-estructura, 2) normas para evaluar la acción de los agentes y 3) la capacidad de atracción y actitudes con respecto a los objetos [40].

La Teoría OCC, ha sido utilizada con éxito para crear modelos que impliquen emociones en el proceso de toma de decisiones. Para mayor información revisar: [22, 24, 26, 36].

\subsection{Diseño del modelo de valoración}

A partir de un análisis, se obtuvo un modelo mental centrado en la evaluación del estado del tablero en curso, mientras se está jugando una partida. 
El modelo mental es una representación del conocimiento procedimental que implica al mundo exterior y que permite deducir información acerca de ese mundo y elegir cursos de acción [15]. En el pseudocódigo de la Figura 3 , se representa el modelo mental del caso de estudio. A partir de éste se determinaron las metas que el agente persigue durante la partida y los acontecimientos que afectan a estas metas.

Las metas perseguidas por el agente, las relaciones entre ellas y los acontecimientos presentes en el tablero son representadas por medio de un diagrama denominado macro-estructura de valoración emotiva. A partir del diagrama se determinan los aspectos del entorno que afectan al cumplimiento de esas metas. Posteriormente se especifica cómo la presencia de estos aspectos modifican la magnitud de las variables que componen la intensidad de la emoción, estas variables están definidas en la teoría OCC.

De esta manera se modela cómo se obtiene la intensidad de la emoción. En la Figura 4 se encuentra la macro-estructura de valoración emotiva obtenida para este proyecto. La emoción que se maneja es la de aliviomiedo: el rango de la intensidad de esta emoción incluye valores positivos y negativos, siendo alivio y miedo respectivamente los puntos extremos de ese rango.

Las variables necesarias para lograr inferir la emoción, a partir de su relación con los acontecimientos del entorno, son la deseabilidad y la excitación, definidas en [40].

\subsection{Mapa cognitivo difuso}

Para representar las relaciones presentes en la macro-estructura de valoración emotiva y cuantificar los efectos de los elementos del entorno, se utilizó un mapa cognitivo difuso (MCD). El MCD es una estructura gráfica difusa, cuyos nodos representan conceptos variables, tales como la desestabilización social en un país o el control político sobre un área geográfica, y cuyas aristas son relaciones de causalidad entre los nodos que conecta [18].

Tanto los conceptos como las aristas pueden tomar cualquier valor en el rango [-1,1]. Se asignan etiquetas lingüisticas a porciones de ese rango por cada concepto, para obtener una interpretación de su valor en el contexto modelado; e. $g$., de 0 a 0.25 , el valor del concepto $x$ es poco; de 0.25 a 0.5 , el valor del concepto $x$ es algo. Con los valores y las etiquetas de los conceptos y relaciones de causalidad se puede determinar la causalidad entre cualquier par de nodos del MCD, además de las reglas difusas para su interpretación [18].

Los MCD's han sido utilizados con éxito para modelar comportamientos 


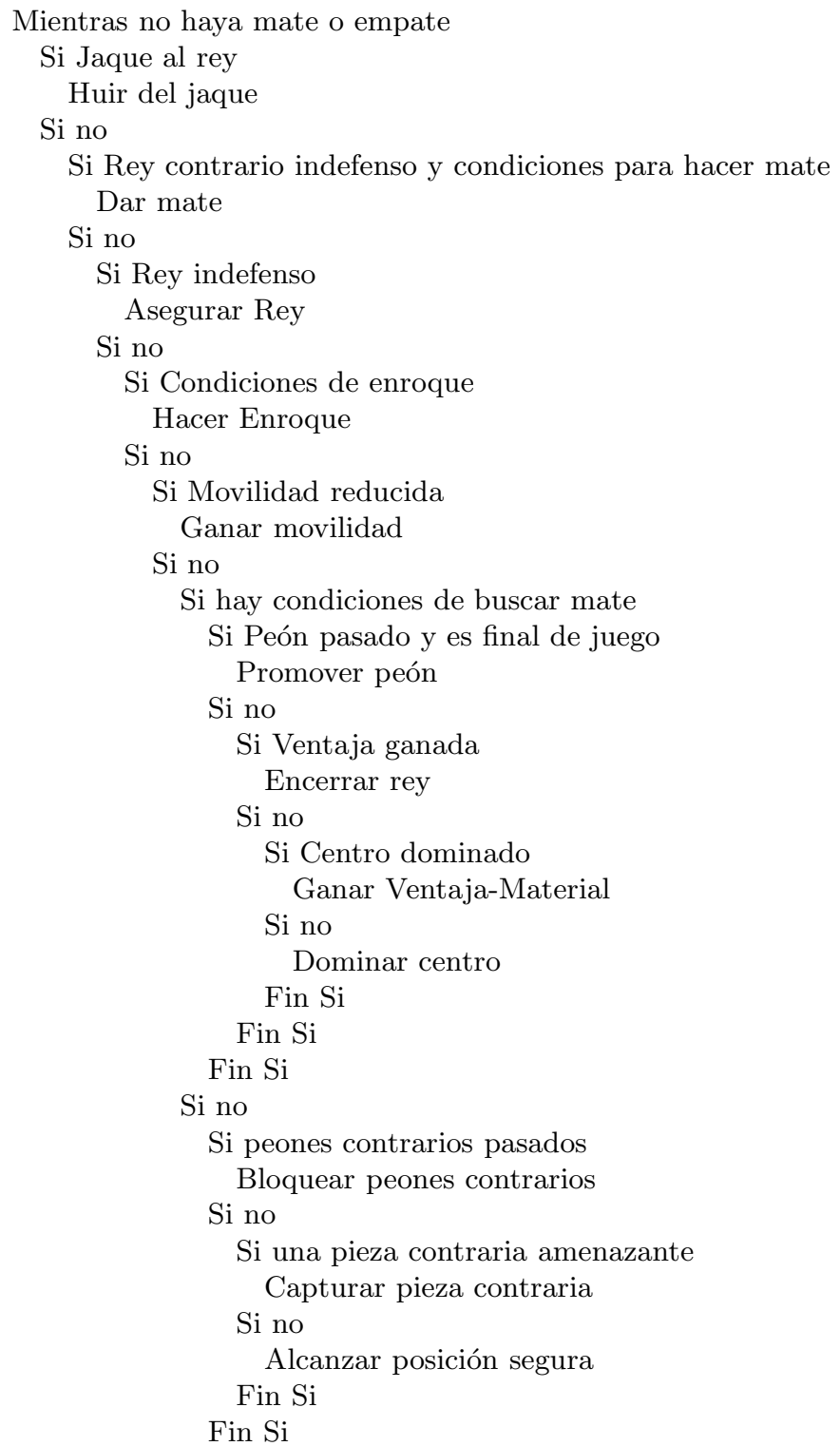

Fin Si

Fin Si

Fin $\mathrm{Si}$

Fin $\mathrm{Si}$

Fin Si

Fin Mientras

Figura 3: Modelo mental del agente cuando está evaluando el tablero. 


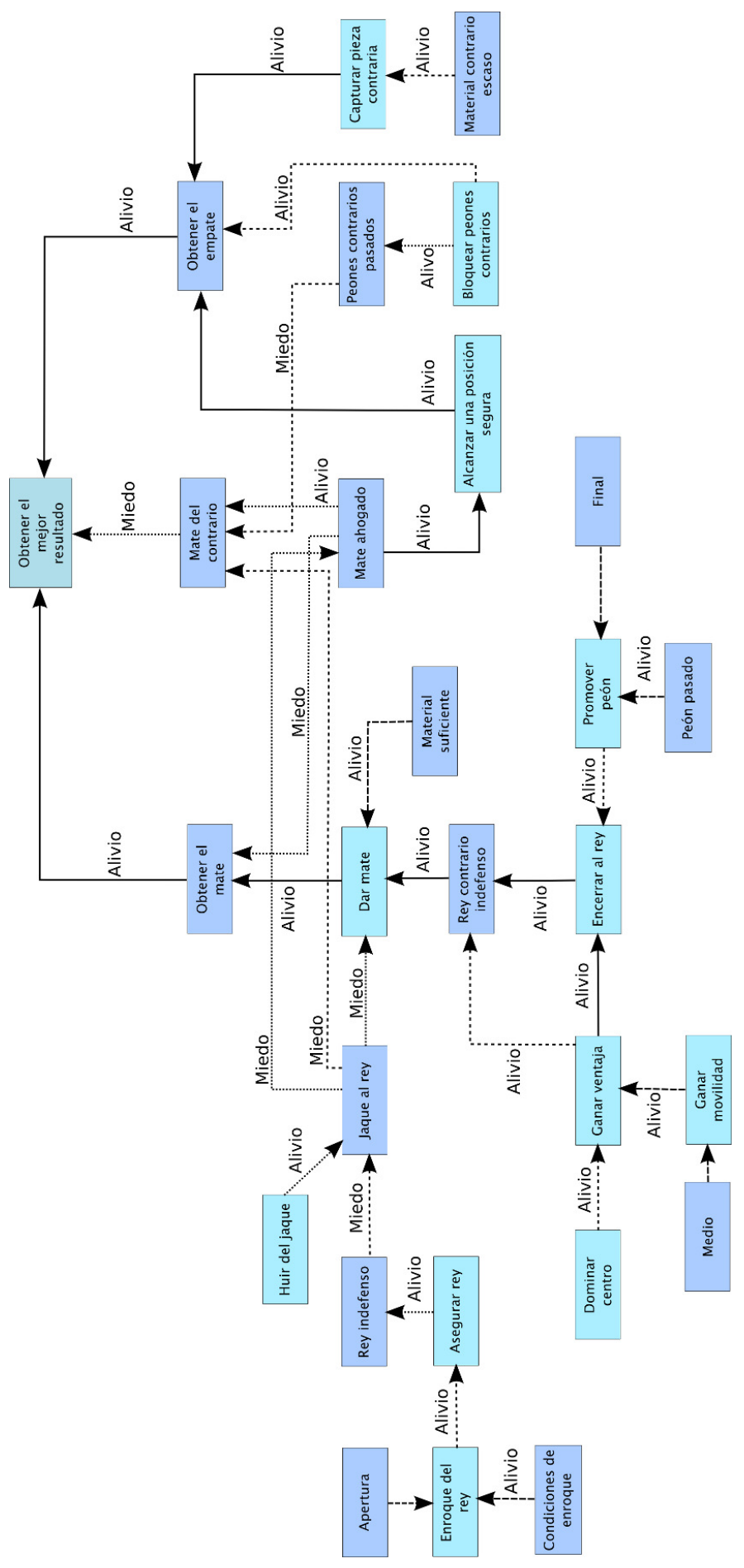

Figura 4: Macro-estructura de valoración. 
humanos [20, 23, 24, 25] y fenómenos físicos [21, 32, 33, 34, 35, 37].

En los MCD's también se puede obtener información del efecto del estado del entorno, representado por los valores asignados a nodos del MCD. Dada la matriz de adyacencia $E$ del MCD, que contiene las causalidades de las aristas que interconectan todos los nodos y un vector de estado $\mathbf{c}_{t}$, que contiene el valor o fuerza de los conceptos de los nodos en el tiempo $t$, tenemos que, en el tiempo $t+1: \mathbf{c}_{t+1}=S\left(\mathbf{c}_{t} \times E\right)$. $S(\mathbf{c})$ es una función umbral no lineal, aplicada de forma individual a cada elemento del vector $\mathbf{c}$ esta función acota la distribución de los valores en el vector de salida $\mathbf{c}_{t+1}$ a partir de los vectores anteriores $\mathbf{c}_{t}$, usándolos en la función $S\left(\mathbf{c}_{t} \times E\right)$. Este proceso se detiene hasta que las fuerzas de los conceptos en $\mathbf{c}_{t}$ converjan en magnitudes similares, es decir, cuando $\mathbf{c}_{t} \approx \mathbf{c}_{t+1}$. Existen varias funciones que pueden utilizarse como función umbral, su selección depende del rango de valores elegido para los conceptos.

Hay dos métodos para interpretar los conceptos. El primer método introduce dos conceptos opuestos en el mismo MCD, e.g.: que en un MCD haya un concepto que se llame decisión errónea y otro que se llame decisión correcta; y que ambos tomen valores en el rango [0,1]. El segundo método de interpretación acepta valores negativos para un concepto, e. g.: que en un MCD haya un concepto llamado decisión, que pueda tomar valores negativos para describir una decisión errónea y valores positivos para una decisión correcta.

Para este proyecto, se eligieron los siguientes conceptos para ser incluidos en el MCD:

1. Conceptos que representan el estado emocional del agente.

(a) La intensidad de la emoción: representa la intensidad de la emoción elicitada en el agente. Cuando la intensidad tiene un valor positivo, representa alivio; cuando tiene uno negativo, representa el miedo. Este concepto tiene valencia, que es la dimensión más importante para diferenciar entre emociones [43].

(b) La deseabilidad: es una variable establecida en la teoría OCC y que está asociada con las reacciones ante acontecimientos del entorno. Se define como el grado en el que un acontecimiento sería deseable o no si ocurriera [40]. Se eligió porque en la macro-estructura se están valorando acontecimientos.

(c) La excitación: es una variable global que también forma parte de la teoría OCC. Se define como la excitación fisiológica que experimenta un individuo [40]. Fue incluida debido a que los jugadores de ajedrez la experimentan durante una partida, ya que este es un deporte bastante competitivo [7, 31]. 


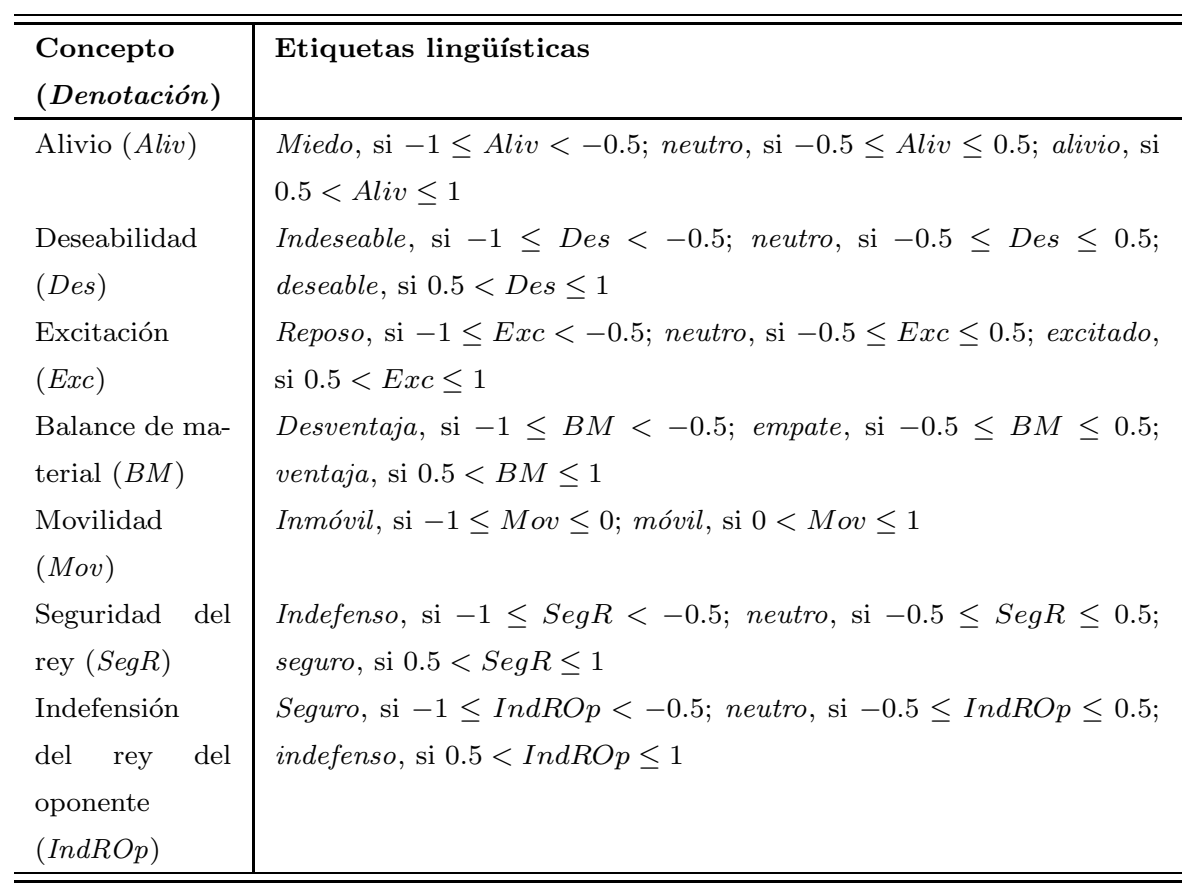

Tabla 1: Conceptos incluidos en el MCD.

2. Parámetros de evaluación del tablero (descritos en la sección 2.2).

(a) El balance de material.

(b) La movilidad.

(c) La seguridad del rey.

(d) La indefensión del rey del oponente, que se mide de la misma manera que el parámetro c), pero con respecto al rey del oponente.

En la Tabla 1 están recopiladas las etiquetas lingüísticas que se les asignaron a cada concepto, así como los valores en los que están divididos sus rangos de valores. La Figura 5 es el MCD que se diseñó tomando en cuenta la macro-estructura de valoración emotiva (Figura 4) y los elementos del entorno (parámetros de evaluación), y su matriz de adyacencia $E$ se encuentra representada en la Tabla 2.

Los valores de causalidad de los enlaces se calibraron para que los elementos del vector de salida convergieran en valores coherentes con el modelo propuesto. La función umbral $S$ elegida es igual a:

$$
S\left(\mathbf{c}_{i}\right)=\frac{2}{1+e^{-5 c_{i}}}-1
$$




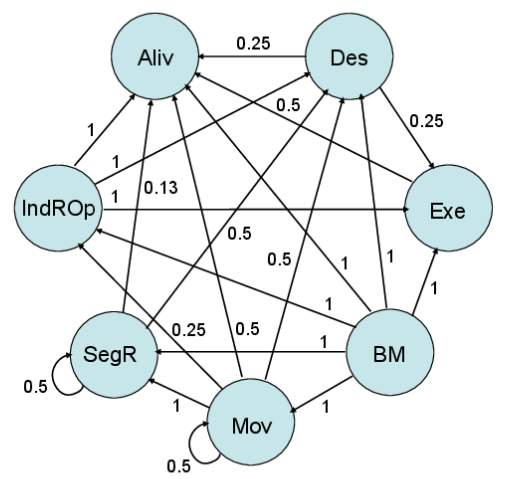

Figura 5: Grafo que representa el mapa cognitivo difuso creado a partir del modelo.

\begin{tabular}{c|ccccccc}
\hline \hline Desde/Hacia & Des & Aliv & Exc & BM & Mov & SegR & IndR Op \\
\hline Des & 0 & 0.25 & 0.25 & 0 & 0 & 0 & 0 \\
Aliv & 0 & 0 & 0 & 0 & 0 & 0 & 0 \\
Exc & 0 & 0.5 & 0 & 0 & 0 & 0 & 0 \\
BM & 1 & 1 & 1 & 0 & 1 & 1 & 1 \\
Mov & 0.5 & 0.5 & 0 & 0 & 0.5 & 1 & 0.25 \\
SegR & 0.5 & 0.13 & 0 & 0 & 0 & 0.5 & 0 \\
IndR Op & 1 & 1 & 1 & 0 & 0 & 0 & 0 \\
\hline \hline
\end{tabular}

Tabla 2: Matriz de adyacencia del mapa cognitivo difuso de la Figura 5. 
donde $c_{i}$ es el valor difuso asignado al nodo $i$. A esta función se le conoce como logística bipolar [19] y ante cualquier valor de entrada en el rango $[-\infty: \infty]$, el valor de salida estará acotado entre [-1:1], que es el rango utilizado para representar la fuerza de cada concepto en el MCD.

La función logística bipolar fue elegida debido a que, dentro de la caracterización del comportamiento, deseamos que la fuerza de cada concepto tenga cambios acelerados cuando el valor de entrada se encuentre en el rango medio, y que además presente saturación cuando el valor de entrada se mueva hacia los valores extremos del rango [41]. Cuando los valores del concepto se alejen del rango utilizado para la representación, deseamos que se les de el mayor peso del rango al que pertenece cada concepto.

\section{Acoplamiento entre el modelo cognitivo de va- loración emotiva y la función de evaluación de tableros}

\subsection{Función de evaluación}

Tal como se menciona en la sección 1, la información extraída por el sistema emotivo de un ser humano, y que es complementaria a la que recogen sus sentidos del entorno, provoca que enfoque su atención sobre ciertos aspectos del mismo entorno: aquellos que tienen mayor relevancia para el cumplimiento de sus metas más urgentes [9, 29]. Debido a dicho efecto, el individuo les da más peso a estos aspectos durante el proceso de toma de decisiones. Es necesario reformular la función de evaluación para que, de acuerdo a la meta activa en el agente y a la intensidad de la emoción determinada por el modelo cognitivo, la magnitud de los parámetros se incremente o decremente, de tal manera que tengan más peso en ella. La función de evaluación del tablero se plantea en la siguiente ecuación:

$$
f_{\text {eval }}\left(P, \varepsilon, R_{\text {Act }}\right)=\sum_{i=1}^{n}\left(1+r_{i} \varepsilon\right) p_{i}
$$

donde: $P$ es el conjunto de los $n$ parámetros de evaluación del tablero $p_{i}$, mencionados en la subsección $2.2 ; \varepsilon$ es la intensidad de la emoción en el agente, que es uno de los nodos del MCD (subsección 3.3); $R_{\text {Act }}$ es un conjunto de factores $r_{i}$, a los cuales se denominó como factores de relevancia, y que están asociados a la meta activa en el agente. A través de estos factores se modifica el peso de cada parámetro en la función de evaluación de acuerdo a la meta activa y a la intensidad de la emoción obtenida en el MCD. 


\begin{tabular}{c|ccc}
\hline \hline $\begin{array}{c}\text { Parámetro de } \\
\text { evaluación }\end{array}$ & $\begin{array}{c}\text { Límite } \\
\text { superior }\left(p_{\max }\right)\end{array}$ & $\begin{array}{c}\text { Límite inferior } \\
\left(p_{\min }\right)\end{array}$ & $\begin{array}{c}\text { Magnitud del } \\
\text { rango }(\operatorname{ran} p)\end{array}$ \\
\hline $\begin{array}{c}\text { Balance de } \\
\text { material }\end{array}$ & 4075 & -4075 & 8150 \\
$\begin{array}{c}\text { Movilidad } \\
\text { Seguridad del rey } \\
\text { Indefensión del } \\
\text { rey del oponente }\end{array}$ & 137 & 0 & 137 \\
Total & 64 & -115.5 & 179.5 \\
\hline \hline
\end{tabular}

Tabla 3: Límites de los rangos de valores de los parámetros de evaluación del tablero.

\subsection{Estimación de los factores de relevancia}

El factor de relevancia representa el efecto de la deformación de la evaluación debido al estado emocional. A cada meta activa se le asocian factores de relevancia para cada parámetro de evaluación. Para estimar un factor de relevancia $r_{i}$, se establece la proporción $\eta_{i}$ deseada para el parámetro de evaluación $p_{i}$ en la función de evaluación; $\eta_{i}$ es equivalente a la ecuación (8):

$$
\eta_{i}=\frac{\operatorname{ran} p_{i}}{\sum^{n} \operatorname{ran} p_{i}} .
$$

$\operatorname{ran} p_{i}$ denota el rango del parámetro $p_{i}$. Se estimó para cada parámetro $p_{i}$ los límites de su rango de valores (Tabla 3 )

La magnitud real del rango de un parámetro es denotado como $\operatorname{ran}_{0} p$; este rango se incrementará o reducirá a un valor final $\operatorname{ran}_{f} p$. Tenemos que:

$$
\operatorname{ran}_{f} p=\operatorname{ran}_{0} p(1+r \varepsilon)
$$

Donde $r$ es el factor de relevancia y $\varepsilon$ es la intensidad de la emoción. Para estimar $r$, se despeja de la ecuación (9):

$$
r=\frac{\frac{\operatorname{ran}_{f} p}{\operatorname{ran}_{0} p}-1}{\varepsilon} .
$$

Obteniendo el rango deseado $\operatorname{ran}_{f} p_{i}$ a partir de (8), y sustituyéndola en (10), tenemos que:

$$
r_{i}=\frac{\frac{\eta_{i} \sum_{j=1}^{n} \operatorname{ran}_{0} p_{j}}{\operatorname{ran}_{0} p_{i}}-1}{\varepsilon} .
$$

Para cada meta activa se establecen las proporciones deseadas para cada parámetro en un valor de intensidad de la emoción, y usando (11), se 


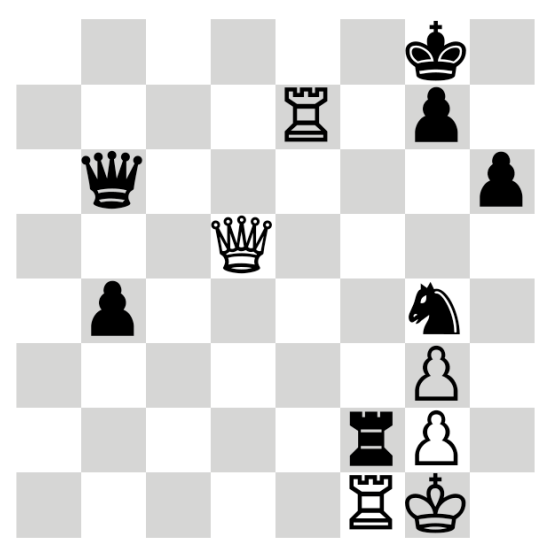

Figura 6: Posición del tablero del problema [39, pág. 8].

estiman los factores de relevancia con los que se obtendrán dichas proporciones.

\section{Pruebas del modelo}

\subsection{Mediciones de los parámetros de evaluación en los nodos terminales de un árbol de juego}

En la primera prueba se usó la posición del tablero representada en la Figura 6.

Esta posición es analizada en [39, pág. 8]. Se asumió que el programa estaba jugando con las piezas negras, cuyo rey se encuentra en jaque. De acuerdo a ese contexto, se estableció que la meta activa del agente es Huir del jaque. De todo el conjunto de jugadas posibles para salir del jaque, hemos elegido 3 para el análisis de esta posición: Kh8, Kf8 y $\mathrm{Kh} 7^{1}$. De acuerdo a [39], la jugada más débil es Kh7, ya que conduce a un empate; Kh8 es la jugada intermedia, ya que las blancas pueden encontrar un contraataque y forzar a un mate ahogado; Kf8 es la jugada más fuerte ya que forza a un triunfo para las piezas negras.

Para esta prueba, se estimó un árbol de juego de 3 niveles de profundidad, el cual está representado en la Figura 7.

\footnotetext{
${ }^{1}$ Las jugadas están denotadas de forma algebraica. La primera letra se refiere a la pieza, la segunda a la columna y el número a la fila. Esta notación está descrita en el reglamento del juego de la Federación Internacional de Ajedrez (FIDE), apéndice E.
} 


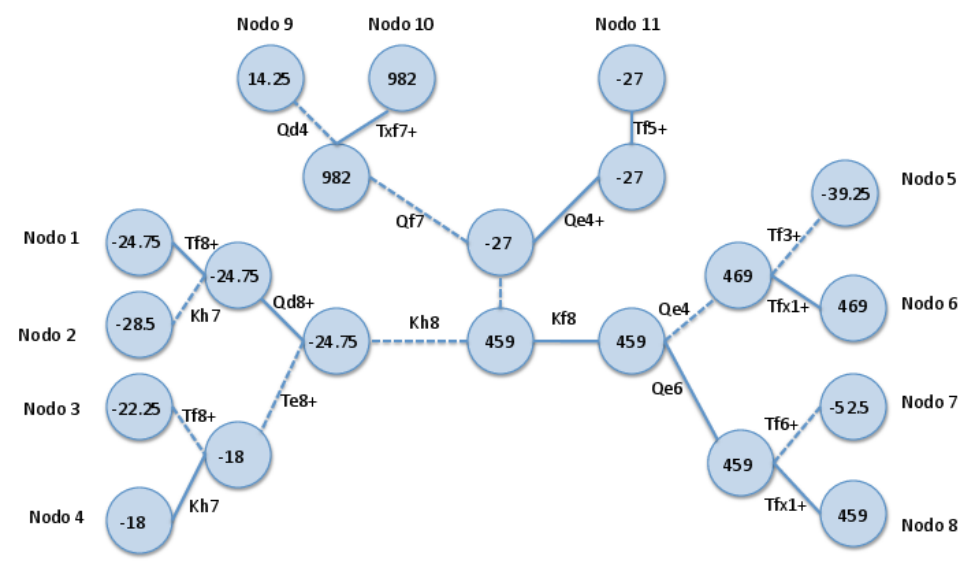

Figura 7: Árbol de juego desarrollado desde la posición del tablero de la Figura 6.

En éste sólo se incluye un subconjunto de todas las jugadas posibles, con el fin de facilitar el análisis. Los cuatro parámetros se midieron en cada posición terminal del árbol, de acuerdo a la forma descrita en la subsección 2.3; estos valores están recopilados en la Tabla 4. La jugada seleccionada de acuerdo a estos valores es Kf8.

\begin{tabular}{c|ccccc}
\hline \hline Nodo & B. M. & Mov. & Seg. R. & Ind. R. Op. & Total \\
\hline 1 & -75 & 35 & -28.75 & -44 & -24.75 \\
2 & -75 & 37 & -36 & -45.5 & -28.5 \\
3 & -75 & 34 & -25.25 & -44 & -22.25 \\
4 & -75 & 38 & -26.5 & -45.5 & -18 \\
5 & -75 & 39 & -48.5 & -45.25 & -39.25 \\
6 & 425 & 40 & -43.25 & -47.25 & 469 \\
7 & -75 & 29 & -51 & -44.5 & -52.5 \\
8 & 425 & 34 & -45.75 & -45.75 & 459 \\
9 & -75 & 37 & -26.75 & -50.5 & -14.25 \\
10 & 900 & 37 & 0.75 & -44.25 & 982 \\
11 & -75 & 30 & -26.75 & -44.75 & -27 \\
\hline \hline
\end{tabular}

Tabla 4: Resultados de la función de evaluación. 


\subsection{Estimación de la intensidad de la emoción a partir del MCD}

De acuerdo al contexto de la posición del tablero (Figura 6), el vector de estado de entrada hacia el MCD que representa el escenario de prueba, es el siguiente:

\begin{tabular}{c|cccccc}
\hline \hline Des & Aliv & Exc & BM & Mov & $\begin{array}{c}\text { SegR } \\
\text { Mio }\end{array}$ & $\begin{array}{c}\text { IndR } \\
\text { Op }\end{array}$ \\
\hline 0 & 0 & 0 & 0 & -1 & -1 & -1 \\
\hline \hline
\end{tabular}

El vector de estado de salida obtenido, que representa el posible escenario resultante del modelo en el MCD, es el siguiente:

\begin{tabular}{c|cccccc}
\hline \hline Des & Aliv & Exc & BM & Mov & $\begin{array}{c}\text { SegR } \\
\text { Mio }\end{array}$ & $\begin{array}{c}\text { IndR } \\
\text { Op }\end{array}$ \\
\hline- & - & - & 0.00000 & - & - & 0.71041 \\
0.97226 & 0.98654 & 0.54248 & 0.99524 & 0.00000 \\
\hline \hline
\end{tabular}

La corrida del escenario de prueba que se realizó puede consultarse en la Tabla 5 .

La intensidad de la emoción corresponde al campo Alivio del vector de salida, que tiene una magnitud de -0.98654; este valor fue introducido en la ecuación (7) para obtener el nuevo valor de utilidad en cada nodo del árbol de la Figura 7.

\subsection{Resultados de la función de evaluación con la intensidad de la emoción}

Con respecto a la meta Huir del jaque se propuso, para probar este modelo, que el parámetro con mayor relevancia fuera la seguridad del rey, seguido en importancia por la movilidad, y por último estarían el balance de material y la indefensión del rey del oponente. Las proporciones de prueba elegidas para estas variables fueron: balance de material $=0.05$, movilidad $=0.3$, seguridad del rey $=0.6$, indefensión del rey del oponente $=0.05$; se propuso que estas proporciones se presentaran cuando la intensidad de la emoción tuviera un valor de -0.7, que corresponde a la sensación de miedo. Usando estas proporciones, los factores de relevancia obtenidos de acuerdo al criterio descrito en la subsección 4.2 fueron los siguientes: balance de material $=1.35$, movilidad $=-25.62$, seguridad del rey $=-39.86$, indefensión del rey del oponente $=-2.01$.

Haciendo uso de estos factores en la ecuación (7), se obtuvieron para cada parámetro las magnitudes que están mostradas en la Tabla 6. 


\begin{tabular}{|c|c|c|c|c|c|c|c|}
\hline $\begin{array}{c}\text { Vector } \\
\text { de } \\
\text { salida }\end{array}$ & Des & Aliv & Exc & BM & Mov & $\begin{array}{l}\text { SegR } \\
\text { Mio }\end{array}$ & IndR Op \\
\hline $\mathrm{V}[0]:$ & 0.000000 & 0.000000 & 0.000000 & 0.000000 & -1.000000 & -1.000000 & 0.000000 \\
\hline $\mathrm{V}[1]:$ & -0.986614 & -0.917817 & 0.000000 & 0.000000 & -0.848284 & -0.998894 & 0.000000 \\
\hline $\mathrm{V}[2]:$ & -0.980447 & -0.964143 & -0.548780 & 0.000000 & -0.785800 & -0.997634 & 0.000000 \\
\hline $\mathrm{V}[3]:$ & -0.977108 & -0.989141 & -0.546081 & 0.000000 & -0.754038 & -0.996758 & 0.000000 \\
\hline $\mathrm{V}[4]:$ & -0.975186 & -0.988114 & -0.544614 & 0.000000 & -0.736391 & -0.996193 & 0.000000 \\
\hline $\mathrm{V}[5]:$ & -0.974045 & -0.987501 & -0.543769 & 0.000000 & -0.726128 & -0.995836 & 0.000000 \\
\hline $\mathrm{V}[6]:$ & -0.973356 & -0.987131 & -0.543266 & 0.000000 & -0.720007 & -0.995614 & 0.000000 \\
\hline $\mathrm{V}[7]:$ & -0.972935 & -0.986904 & -0.542963 & 0.000000 & -0.716302 & -0.995475 & 0.000000 \\
\hline $\mathrm{V}[8]:$ & -0.972678 & -0.986765 & -0.542777 & 0.000000 & -0.714040 & -0.995389 & 0.000000 \\
\hline $\mathrm{V}[9]:$ & -0.972519 & -0.986679 & -0.542664 & 0.000000 & -0.712651 & -0.995336 & 0.000000 \\
\hline $\mathrm{V}[10]:$ & -0.972421 & -0.986626 & -0.542594 & 0.000000 & -0.711795 & -0.995303 & 0.000000 \\
\hline $\mathrm{V}[11]:$ & -0.972361 & -0.986594 & -0.542551 & 0.000000 & -0.711267 & -0.995282 & 0.000000 \\
\hline $\mathrm{V}[12]:$ & -0.972323 & -0.986573 & -0.542524 & 0.000000 & -0.710941 & -0.995270 & 0.000000 \\
\hline $\mathrm{V}[13]:$ & -0.972300 & -0.986561 & -0.542507 & 0.000000 & -0.710739 & -0.995262 & 0.000000 \\
\hline $\mathrm{V}[14]:$ & -0.972286 & -0.986553 & -0.542497 & 0.000000 & -0.710615 & -0.995257 & 0.000000 \\
\hline $\mathrm{V}[15]:$ & -0.972277 & -0.986548 & -0.542491 & 0.000000 & -0.710537 & -0.995254 & 0.000000 \\
\hline $\mathrm{V}[16]:$ & -0.972271 & -0.986545 & -0.542487 & 0.000000 & -0.710489 & -0.995252 & 0.000000 \\
\hline $\mathrm{V}[17]:$ & -0.972268 & -0.986544 & -0.542485 & 0.000000 & -0.710460 & -0.995251 & 0.000000 \\
\hline $\mathrm{V}[18]:$ & -0.972266 & -0.986542 & -0.542483 & 0.000000 & -0.710442 & -0.995250 & 0.000000 \\
\hline $\mathrm{V}[19]:$ & -0.972265 & -0.986542 & -0.542482 & 0.000000 & -0.710430 & -0.995250 & 0.000000 \\
\hline $\mathrm{V}[20]:$ & -0.972264 & -0.986541 & -0.542482 & 0.000000 & -0.710423 & -0.995250 & 0.000000 \\
\hline $\mathrm{V}[21]:$ & -0.972263 & -0.986541 & -0.542481 & 0.000000 & -0.710419 & -0.995249 & 0.000000 \\
\hline $\mathrm{V}[22]:$ & -0.972263 & -0.986541 & -0.542481 & 0.000000 & -0.710416 & -0.995249 & 0.000000 \\
\hline $\mathrm{V}[23]:$ & -0.972263 & -0.986541 & -0.542481 & 0.000000 & -0.710414 & -0.995249 & 0.000000 \\
\hline $\mathrm{V}[24]:$ & -0.972263 & -0.986541 & -0.542481 & 0.000000 & -0.710413 & -0.995249 & 0.000000 \\
\hline $\mathrm{V}[25]:$ & -0.972263 & -0.986541 & -0.542481 & 0.000000 & -0.710413 & -0.995249 & 0.000000 \\
\hline
\end{tabular}

Tabla 5: Corrida del mapa cognitivo. 


\begin{tabular}{c|ccccc}
\hline \hline Nodo & BM & Mov & SegR & IndR Op & Total \\
\hline 1 & 25.09 & 919.57 & -1159.23 & -131.33 & -83.23 \\
2 & 25.09 & 972.12 & -1451.56 & -135.81 & -318.53 \\
3 & 25.09 & 893.3 & -1018.11 & -131.33 & 31.62 \\
4 & 25.09 & 998.4 & -1068.51 & -135.81 & 90.79 \\
5 & 25.09 & 1024.67 & -1955.57 & -135.06 & -770.75 \\
6 & -142.2 & 1050.94 & -1743.89 & -141.03 & -694.11 \\
7 & 25.09 & 761.93 & -2056.38 & -132.83 & -1136.52 \\
8 & -142.2 & 893.3 & -1844.69 & -136.56 & -957.03 \\
9 & 25.09 & 972.12 & -1078.59 & -150.74 & 69.36 \\
10 & -301.13 & 972.12 & 30.24 & -132.08 & 833.31 \\
11 & 25.09 & 788.21 & -1078.59 & -133.57 & -131.72 \\
\hline \hline
\end{tabular}

Tabla 6: Resultados de la función de evaluación afectada por la emoción.

Siguiendo estos resultados, después de correr el algoritmo de búsqueda se eligió una rama distinta a la que se había elegido previamente y que corresponde a la jugada Kh8 (Figura 7). En el análisis realizado en [39], Kh8 corresponde a una jugada que es defensiva, y que puede conducir a un empate por parte del oponente si éste responde de manera correcta.

Esta elección es congruente con el efecto de tomar una decisión que dé mayor prioridad a la seguridad del rey, en lugar de elegir la jugada más ofensiva que es Kf8.

\section{Conclusiones}

El objetivo de este trabajo es el desarrollo de un agente computacional llamado Deep Feeling, que juega ajedrez de acuerdo a la reacción emocional que podría sentir un jugador en instantes durante la partida [11]. En el proceso de toma de decisiones, la intensidad del estado emocional del individuo influye en la evaluación de consecuencias de una decisión [28].

Este trabajo representa el análisis y diseño del motor de inferencia de Deep Feeling, cuyo fin es incorporar emociones en el proceso de toma de decisiones. Este motor es utilizado para afectar la función de evaluación de un programa que juegue ajedrez. Para lograrlo, se han incluido aspectos del estado emocional en la función de evaluación, representados por el mapa cognitivo difuso. La propuesta básica consiste en cambiar de magnitud los pesos de los parámetros de evaluación del tablero de acuerdo a la intensidad de la emoción y a la meta activa: esto implica un cambio en la relevancia de éstos y en el resultado de la función de evaluación, lo que a su vez permite que la elección de la siguiente jugada sea acorde a esos 
pesos. Pretendemos hacer congruente la elección con el fenómeno de la influencia del estado emocional en el proceso de toma de decisiones: esta es la inspiración principal de este trabajo.

Como parte de los resultados, se tiene el prototipo implementado de Deep Feeling. Se hicieron pruebas con el programa y hay un desplazamiento en el espacio de búsqueda que permite que el agente computacional elija una jugada distinta. Las jugadas obtenidas cumplen con los propósitos establecidos para las metas bajo las cuales fueron elegidas. Para mayor información consultar $[11,12,13]$.

Los seres humanos no podemos calcular frente a un evento todas las posibles salidas. Las emociones tienen una función importante en ese sentido, ya que nuestro espacio de búsqueda se restringe a lo que realmente es importante para nuestras metas: las emociones son un mecanismo de interrupción que ayuda a enfocar nuestros recursos mentales y físicos en lo que es relevante para nosotros $[9,45]$. La incorporación de esta fenomenología a la computación forma parte de una línea de investigación denominada computación afectiva [41]; con este trabajo, pretendemos hacer una contribución al análisis y diseño de emociones sintéticas.

Reconocimientos: este trabajo forma parte de la investigación desarrollada por Diego Enrique Hernández-González [12,13]. para obtener el grado de Maestro en Ciencias en el Posgrado en Ciencia e Ingeniería de la Computación de la Universidad Nacional Autónoma de México, CVU 225723. Además forma parte del proyecto Computación Suave y Aplicaciones, financiado por la Universidad Autónoma Metropolitana (http://ce.azc.uam.mx/profesores/clc/). Durante el año de 2010 Martha Mora-Torres estuvo como Profesor visitante en la misma Universidad.

\section{Referencias}

[1] Acevedo-Moreno, D. A. (2009) Diseño de una Arquitectura para Incorporar Emociones en Videojuegos. Tesis de maestría, Universidad Nacional Autónoma de México, Ciudad de México. Recuperado de http://ce.azc.uam.mx/profesores/clc/.

[2] Acevedo-Moreno, D. A. (2011) "Diseño de una arquitectura para incorporar emociones en un videojuego", Revista Ciencia, Tecnología e Innovación para el Desarrollo de México, sección Tesis de Posgrado: A4-0011-DF -2009-MT. En línea: http://pcti.mx. 
[3] Ariely, D.; Loewenstein, G. (2006) "The heat of the moment: the effect of sexual arousal on sexual decision making", J. Behav. Decis. Making 19(2): 87-98.

[4] Beal, D. F.; Smith, M. C. (1997) "Learning piece values using temporal differences", International Computer Chess Association Journal 20(3): $147-151$.

[5] Charness, N.; Tuffiash, M.; Jastrzembski, T. (2004) "Motivation, emotion, and expert skill acquisition", en: Dai, D. Y.; Sternberg, R. J. (Eds.) Motivation, Emotion, and Cognition: Integrative Perspectives on Intellectual Functioning and Development, Lawrence Erlbaum Associates, Estados Unidos: 299-320.

[6] Degroot, D.; Broekens, J. (2003) "Using negative emotions to impair gameplay", en: Heskes, T. M., Lucas, P. J. F., Vuurpijl, L. G., y Wiegerinck, W. A., (Eds.) 15th Belgium-Netherlands Artificial Intelligence Conference, Nijmegen, Holanda.

[7] Fine, R. (1974) Psicología del Jugador de Ajedrez. Ediciones Martínez Roca, Barcelona, España.

[8] Frey, P. W. (1977) "An introduction to computer chess", en: Frey, P. W. (Ed.) Chess Skill in Man and Machine, Texts and Monographs in Computer Science, Springer-Verlag, Estados Unidos: 54-81.

[9] Hanoch, Y. (2002) "Neither an angel nor an ant: Emotion as an aid to bounded rationality", Journal of Economic Psychology 23(1): 1-25.

[10] Heisman, D. (2003) "Evaluation criteria", en: http://www . chesscafe.com/text/heisman27.pdf, consultado el 27/10/2011, $15: 24$.

[11] Hernández-González, D. E.; Laureano-Cruces, A. L. (2009) "Influencia de las emociones durante una partida de ajedrez", en: Avances en Tecnologías de la Información: Memorias del XXII Congreso Nacional y VIII Congreso Internacional de Informática y Computación de la ANIEI, Alfaomega Grupo Editor, Ensenada, Baja California: 343-353.

[12] Hernández-González, D. E. (2011a) Influencia de las Emociones en el Proceso de Toma de Decisiones. Tesis de Maestría, Universidad Nacional Autónoma de México, Ciudad de México. Recuperado de http://ce.azc.uam.mx/profesores/clc/. 
[13] Hernández-González, D. E. (2011b) "Influencia de las emociones en el proceso de toma de decisiones", Revista Ciencia, Tecnología e Innovación para el Desarrollo de México, sección Tesis de Posgrado: A4-0014-DF -2011-MT. En linea: http://pcti.mx.

[14] Horgan, D. D. (1992) "Children and chess expertise: the role of calibration", Psychological Research 54(1):44-50.

[15] Johnson-Laird, P. N. (2005) "Mental models and thought", en: Holyoak, K. J. \& Morrison, R. G. (Eds.) The Cambridge Handbook of Thinking and Reasoning, Cambridge University Press: 185-208.

[16] Joireman, J.; Fick, C.; Anderson, J. (2002) "Sensation seeking and involvement in chess", Personality and Individual Differences 32(3): 509-515.

[17] Jones, L. T.; Rey, P. D.; Howden, A.; Knighton, M. S.; Kittinger, D. L.; Hollander, R. E. (1983) "Robot computer chess game", U. S. Patent 4398720 .

[18] Kosko, B. (1986) "Fuzzy cognitive maps", International Journal of Man-Machine Studies 24(1): 65-75.

[19] Kosko, B. (1992) Neural Networks and Fuzzy Systems : a Dynamical Systems Approach to Machine Intelligence. Prentice Hall.

[20] Laureano-Cruces, A. L.; Ramírez-Rodríguez, J.; Terán-Gilmore, A. (2004) "Evaluation of the teaching-learning process with fuzzy cognitive maps", en: Lemaître, C.; Reyes, C. A. \& González, J. A. (Eds.) Advances in Artificial Intelligence - IBERAMIA 2004, Lecture Notes in Computer Science 3315, Springer Verlag: 922-931.

[21] Laureano-Cruces, A. L.; Ramírez-Rodríguez, J.; Mora-Torres, M.; Espinosa-Paredes, G. (2006) "Modeling a risk scenario: small LOCA in a nucleoelectric plant using fuzzy cognitive maps", Research in Computing Science 26: 3-13.

[22] Laureano-Cruces, A. L.; Mora-Torres, M.; Ramírez-Rodríguez, J.; Gamboa-Rodríguez, F. (2009) "Emotions as an element that maximizes the effectiveness of a pedagogical agent", en: E-Learn 2009: World Conference on E-Learning in Corporate Govermment, Healthcare, $\mathcal{G}$ Higher Education, Association for the Advancement of Computing in Education, Vancouver, Canada: 2817-2822. 
[23] Laureano-Cruces, A. L.; Ramírez-Rodríguez, J.; Mora-Torres, M.; de Arriaga, F.; Escarela-Pérez, R. (2010) "Cognitive-operative model of intelligent learning systems behavior", Interactive Learning Environments, 18(1): 11-38.

[24] Laureano-Cruces, A.L.; Rodríguez-García, A. (2011) "Design and implementation of an educational virtual pet using the OCC theory", Journal of Ambient Intelligence and Humanized Computing 3(1): $61-71$.

[25] Laureano-Cruces, A. L.; Guadarrama-Ponce, C.; Mora-Torres, M.; Ramírez-Rodríguez, J. (2011) "A cognitive model for the red baron: a perspective taking into account emotions", ICGST-Artificial Intelligence Machine Learning Journal 11(2): 5-13.

[26] Laureano-Cruces, A. L.; Mora-Torres, M.; Ramírez-Rodríguez, J.; De Arriaga-Gómez, F. (2011) "Operative strategies related to an affective-motivational architecture to achieve instructional objectives", ICGST-Artificial Intelligence Machine Learning Journal 11(2): 15-20.

[27] Leite, I.; Martinho, C.; Pereira, A.; Paiva, A. (2008) "iCat: an affective game buddy based on anticipatory mechanisms", en: AAMAS '08: Proceedings of the 7th International Joint Conference on Autonomous Agents and Multiagent Systems, International Foundation for Autonomous Agents and Multiagent Systems, Richland, Escocia: 1229-1232.

[28] Loewenstein, G.; Lerner, J. S. (2003) "The role of affect in decision making", en: Davidson, R. J.; Goldsmith, H. H. \& Scherer, K. R. (Eds.) Handbook of Affective Science, Series in Affective Science, Oxford University Press, Nueva York: 619-642.

[29] Loewenstein, G. F.; Weber, E. U.; Hsee, C. K.; Welch, N. (2001) "Risk as feelings", Psychological Bulletin 127(2): 267-286.

[30] Marsland, T. A. (1992) "Computer Chess and Search", en: Shapiro, S. C. (Ed.) Encyclopedia of Artificial Intelligence, 2da. edición, John Wiley \& Sons Ltd., New Jersey, Estados Unidos.

[31] Mazur, A.; Booth, A.; Dabbs, J. M. (1992) "Testosterone and Chess Competition", Social Psychology Quarterly 55(1): 70-77.

[32] Méndez-Gurrola, I. I. (2007) Sistema basado en el conocimiento para la predicción de los efectos de una supernova en el medio interestelar. 
Tesis de Maestría, Universidad Autónoma Metropolitana, Ciudad de México. Recuperado de http://ce.azc.uam.mx/profesores/clc/.

[33] Méndez-Gurrola, I. I.; Laureano-Cruces, A. L.; Santillán-González, A. J.; Ramírez-Rodríguez, J. (2008) "A knowledge based system design for the prediction of supernova effects in the interstellar environment", en: Gelbukh, A. \& Kuri-Morales A. F. (Eds.) MICAI 2007. IEEE Computer Society Press, pp. 26-35.

[34] Mora-Torres, M. (2007) Sistema Experto en la Toma de Decisiones de un Escenario de Riesgo: L. O. C. A. Pequeño en una Planta Nucleoeléctrica. Tesis de Maestría, Universidad Nacional Autónoma de México, Ciudad de México. Recuperado de http://ce.azc.uam.mx/ profesores/clc/.

[35] Mora-Torres, M.; Laureano-Cruces, A. L.; Ramírez-Rodríguez, J.; Espinosa-Paredes, G. (2009) "Analysis and design of the knowledge representation for the implementation of a distributed reasoning", Revista de Matemática: Teoría y Aplicaciones 16(2): 188-202.

[36] Mora-Torres, M.; Laureano-Cruces, A. L.; Velasco-Santos, P. (2011) "Estructura de las emociones dentro de un proceso de enseñanza-aprendizaje", Revista Perfiles Educativos-UNAM, XXXIII(131):64-79. Recuperado de http://www.iisue.unam.mx/ seccion/perfiles/.

[37] Mora-Torres, M. (2011) "Sistema experto en la toma de decisiones de un escenario de riesgo: LOCA pequeño en una planta nucleoeléctrica", Revista Ciencia, Tecnología e Innovación para el Desarrollo de México., sección Tesis de Posgrado: A4-0013-DF -2011-MT. En línea: http://pcti.mx.

[38] Newborn, M. (1975) Computer Chess. Association for Computing Machinery Monograph, Academic Press, 1975.

[39] Nunn, J. (2007) Secrets of Practical Chess. Gambit.

[40] Ortony, A.; Clore, G. L.; Collins, A. (1996) La Estructura Cognitiva de las Emociones. Siglo Veintiuno de España Editores, Madrid, España.

[41] Picard, R. W. (1997) Affective Computing. The MIT Press, Boston, Estados Unidos.

[42] Sajó, L.; Ruttkay, Z.; Fazekas, A. (2011) “Turk-2, a multi-modal chess player", International Journal of Human-Computer Studies 60(7-8): 483-495. 
[43] Scherer, K. R. (2000) "Psychological Models of Emotion", en: Borod, J. C. (Ed.) The Neuropsychology of Emotion, Oxford University Press US, Nueva York: 137-166.

[44] Shannon, C. E. (1950) "Programming a computer for playing chess", Philosophical Magazine 41: 256-275.

[45] Simon, H. A. (1967) "Motivational and emotional controls of cognition", Psychological Review 74(1): 29-39.

[46] Slater, E. (1988) "Statistics for the chess computer and the factor of mobility", en: Levy, D. (Ed.) Computer Chess Compendium, Springer-Verlag, Nueva York: 113-115. 
